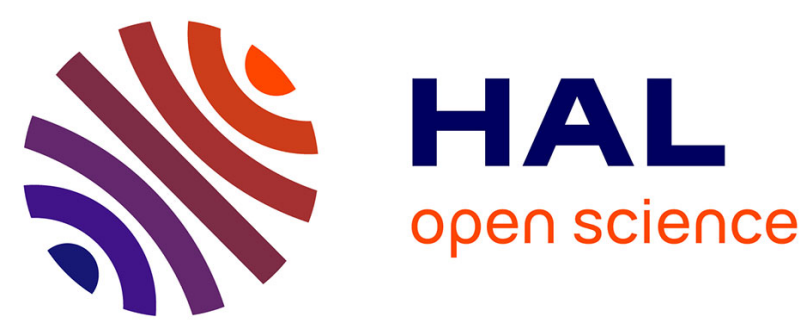

\title{
Bond formation and slow heterogeneous dynamics in adhesive spheres with long-ranged repulsion: Quantitative test of mode coupling theory
}

\author{
O. Henrich, A. M. Puertas, M. Sperl, J. Baschnagel, M. Fuchs
}

\section{- To cite this version:}

O. Henrich, A. M. Puertas, M. Sperl, J. Baschnagel, M. Fuchs. Bond formation and slow heterogeneous dynamics in adhesive spheres with long-ranged repulsion: Quantitative test of mode coupling theory. Physical Review E: Statistical, Nonlinear, and Soft Matter Physics, 2007, 76 (3), pp.031404. 10.1103/PhysRevE.76.031404 . hal-00355304

\author{
HAL Id: hal-00355304 \\ https://hal.science/hal-00355304
}

Submitted on 5 May 2020

HAL is a multi-disciplinary open access archive for the deposit and dissemination of scientific research documents, whether they are published or not. The documents may come from teaching and research institutions in France or abroad, or from public or private research centers.
L'archive ouverte pluridisciplinaire HAL, est destinée au dépôt et à la diffusion de documents scientifiques de niveau recherche, publiés ou non, émanant des établissements d'enseignement et de recherche français ou étrangers, des laboratoires publics ou privés. 


\title{
Bond formation and slow heterogeneous dynamics in adhesive spheres with long-ranged repulsion: Quantitative test of mode coupling theory
}

\author{
O. Henrich, ${ }^{1}$ A. M. Puertas, ${ }^{2}$ M. Sperl, ${ }^{1}$ J. Baschnagel, ${ }^{3}$ and M. Fuchs ${ }^{1}$ \\ ${ }^{1}$ Department of Physics, University of Konstanz, 78457 Konstanz, Germany \\ ${ }^{2}$ Group of Complex Fluids Physics, Department of Applied Physics, University of Almeria, 04120 Almeria, Andalucía, Spain \\ ${ }^{3}$ Institut Charles Sadron, 6, rue Boussingault, BP 40016, 67083 Strasbourg Cedex, France
}

(Received 7 May 2007; published 27 September 2007)

\begin{abstract}
A colloidal system of spheres interacting with both a deep and narrow attractive potential and a shallow long-ranged barrier exhibits a prepeak in the static structure factor. This peak can be related to an additional mesoscopic length scale of clusters and/or voids in the system. Simulation studies of this system have revealed that it vitrifies upon increasing the attraction into a gel-like solid at intermediate densities. The dynamics at the mesoscopic length scale corresponding to the prepeak represents the slowest mode in the system. Using mode coupling theory with all input directly taken from simulations, we reveal the mechanism for glassy arrest in the system at $40 \%$ packing fraction. The effects of the low- $q$ peak and of polydispersity are considered in detail. We demonstrate that the local formation of physical bonds is the process whose slowing down causes arrest. It remains largely unaffected by the large-scale heterogeneities, and sets the clock for the slow cluster mode. Results from mode-coupling theory without adjustable parameters agree semiquantitatively with the local density correlators but overestimate the lifetime of the mesoscopic structure (voids).
\end{abstract}

DOI: 10.1103/PhysRevE.76.031404

PACS number(s): 82.70.Dd, 61.20.Ja, 64.70.Pf

\section{INTRODUCTION}

Many materials solidify into nonequilibrium structures when particle interactions become strong and if crystallization proceeds too slowly. Colloidal dispersions provide a number of model systems to study these still little understood solidification phenomena as particle potentials can be tailored and detailed experimental observations are possible. For example, colloidal hard spheres have been studied intensively, where the addition of a nonadsorbing polymer induces an attraction, whose strength and range can be controlled [1]. The case of short-ranged attractions has turned out especially rich. Colloidal particles interacting with already moderately strong short-ranged attractions can form metastable amorphous solids, which exhibit exceedingly long life times.

Depending on the colloidal density and attraction strength different types of metastable arrested solids can be formed. At high densities, glasses are observed when repulsions hinder and even prevent structural rearrangements [2]. Weak short-ranged attractions at first melt these "repulsion-driven glasses" because they distort and loosen the local packing. At attraction strengths somewhat higher, yet still of the order of the thermal energy, physical bonds are formed in dense systems, which leads to aggregation into "attraction-driven glasses" [3-5]. At attraction strengths high compared to thermal fluctuations aggregation phenomena proceed far from equilibrium at low density, resulting in tenuous solids, i.e., gels. At even lower density, the aggregation process leads to cluster formation and aggregation [6-10].

The connection between repulsion- and attraction-driven glass transitions at high densities has been understood within a microscopic theoretical framework, namely, mode coupling theory (MCT) [11-15]. Yet, the mechanisms of solidification at intermediate attraction strengths and low to intermediate densities are still not completely understood $[16,17]$. Espe- cially the interplay with equilibrium phase transitions and percolation is presently under scrutiny $[7,18,19]$; gelation has been interpreted as phase separation arrested due to vitrification of the liquid phase, leading to independent clusters at low density $[8,20,21]$. Another aspect that has been considered at low densities is the existence of weak long-ranged repulsive interactions ("barriers") between the particles, that may induce density modulations (or nonpercolating clusters at low density), and thus prevent solidification for some parameter ranges [22]. Small wave-vector scattering peaks indicative of mesoscale correlations have also been observed and employed to characterize the cluster or gel structures $[10,23,24]$. Their observation in equilibrium systems with long-ranged barriers is expected because these systems show tendencies to microphase separate and to form interconnected structures of clusters and voids [25]. Yet, such long distance correlations and clusters of finite size were also observed in systems where barriers supposedly are absent and presumably have a nonequilibrium origin and arise from phase separation and/or aggregation and coarsening [8,9]. Nonequilibrium origins of the low angle scattering peaks also were suggested by the observation of their time evolution $[23,26]$. Alternatively, to reach gelation from the fluid, liquid-gas phase separation can be prevented setting a maximum number of bonds per particle [27] or total number of bonds in the system [28]. In these cases, gelation is directly connected to percolation, and the low- $q$ modes facilitate the relaxation of the whole system, due to the lack of stiffness (only two-four neighbors are allowed on average). At higher density, such modes are expected to be less important than collective relaxations, and a general theory should account for both relaxation mechanisms.

In this contribution we test quantitatively the MCT predictions for low density attractive glasses or high density gels and study the role of the cluster or void structure on bond formation at an intermediate density. Previous studies 
have shown that attractive glasses found in simulations have many qualitative properties predicted by MCT [29-32]. Here the input structure factor needed for the MCT calculations is taken from simulations, and we compare the properties of the density correlation functions quantitatively. Additionally, by comparing the results of MCT calculations for systems with and without small wave-vector prepeak in the structure, we highlight the importance of mesoscopic heterogeneities on attraction-driven dynamic arrest. We consider a system of particles interacting with a narrow attraction and a weak long-ranged repulsion whose dynamics has been studied intensely by simulations $[3,31-34]$. At the considered density of $40 \%$ packing fraction, our system is above the percolation threshold, and exhibits an equilibrium structural pre-peak at small wave vectors for parameter ranges where the barrier suppresses phase separation. By switching off the repulsive barrier, the cluster or void structure can be eliminated and the system becomes homogeneous (for polymer fractions below phase separation).

Similar studies, taking the $S_{q}$ from simulations as input for MCT, have been performed previously in repulsiondriven systems, for instance, Lennard-Jones [35,36], molecular glasses [37], silica [38], or sodium silicate melts [39]. For attraction-driven colloidal glasses, a similar study has recently been performed by Manley et al. using experimental structure factors [20], and we reach an analogous conclusion concerning the mechanism of dynamic arrest. Our study goes beyond Ref. [20] because the input to our MCT calculations is taken from simulations of the system we address without adjustable parameters, while Manley et al. adjusted the colloid density arbitrarily and assumed a specific form for the static structure factor at large wave vectors. We find that it is exactly the structure at large wave vectors that dominates bond formation, and elucidate the role of polydispersity and the large scale structures on the dynamics of the system. Similar effects in the dynamics related to a prepeak in $S_{q}$ have also been observed in sodium silicate melts $[39,40]$.

\section{MODE COUPLING THEORY}

This section aims to give a brief overview of the most important results within mode coupling theory (MCT) concerning the description of liquid-to-glass nonergodicity transitions $[41,42]$. We focus on the coherent part of the density correlation function as it provides most insights into the physical mechanisms causing glassy arrest.

MCT gives a self-consistent equation of motion of the (normalized) intermediate scattering function $\Phi_{q}(t)$, which is the coherent part of the autocorrelation function of density fluctuations with wave vector $\mathbf{q}$ of $N$ particles, defined by

$$
\Phi_{q}(t)=\frac{1}{N S_{q i, j=1}} \sum_{i}^{N}\left\langle\exp \left\{i \mathbf{q} \cdot\left[\mathbf{r}_{i}(t)-\mathbf{r}_{j}(0)\right]\right\}\right\rangle .
$$

The normalization to unity at time $t=0$ is provided by the static structure factor $S_{q}=\Sigma_{i, j=1}^{N}\left\langle\exp \left\{i \mathbf{q} \cdot\left[\mathbf{r}_{i}-\mathbf{r}_{j}\right]\right\}\right\rangle / N$, which captures equilibrium density correlations. The equation of motion for $\Phi_{q}(t)$ takes the form of a relaxation equation, where retardation effects with respect to exponential relax- ation on diffusive time scale $\mathcal{T}_{q}=\left(D^{s} q^{2} / S_{q}\right)^{-1}$ are contained in a memory kernel $m_{q}(t)$.

$$
\mathcal{T}_{q} \dot{\Phi}_{q}(t)+\Phi_{q}(t)+\int_{0}^{t} d t^{\prime} m_{q}\left(t-t^{\prime}\right) \dot{\Phi}_{q}\left(t^{\prime}\right)=0 .
$$

The initial decay constant $\mathcal{T}_{q}$ describes short time diffusive particle motion, and is set by the short time collective diffusion coefficient $D^{s}$; it captures instantaneous particle interactions and will not play an important role at the glass transition.

The central quantity capturing slow structural rearrangements close to glassy arrest is the memory function $m_{q}(t)$, which is given in MCT approximation as follows:

$$
m_{q}(t)=\mathcal{F}_{q}[\{\Phi(t)\}]=\frac{1}{2} \sum_{\mathbf{k p}} V_{\mathbf{q k p}} \Phi_{k}(t) \Phi_{p}(t) .
$$

The vertices $V_{\text {qkp }}$ couple density fluctuations of different wavelengths and thereby capture a nonlinear feedback mechanism in dense fluids, which is interpreted as "cage effect" [42]. The memory kernel can be regarded as a generalized friction kernel, as can easily be verified after timeFourier transformation of Eq. (2). MCT is a first principles approach as the vertices are calculated from the microscopic interactions

$$
V_{\mathbf{q k p}}=S_{q} S_{k} S_{p} \frac{\rho^{2}}{N q^{4}}\left[\mathbf{q} \cdot \mathbf{k} c_{k}+\mathbf{q} \cdot \mathbf{p} c_{p}\right]^{2} \delta(\mathbf{q}-\mathbf{k}-\mathbf{p}) .
$$

The mode coupling approximation for $m_{q}$ yields a set of equations that is solved self-consistently. Hereby the only input to the theory is the static equilibrium structure factor $S_{q}$, which enters the memory kernel $m_{q}$ directly and via the direct correlation function $c_{q}=\left(1-1 / S_{q}\right) / \rho$, with $\rho=N / V$ the average density. According to MCT the dynamics of the dense fluid is therefore completely determined by equilibrium quantities plus one scale factor to set the time scale. In the following, we will take $S_{q}$ directly from simulations.

Solutions of Eqs. (2)-(4) show a bifurcation scenario due to the nonlinear nature of the equations. The bifurcation point is identified with an idealized liquid-to-glass transition. A quantity of special interest is the long-time limit of the normalized density correlator, $f_{q}=\lim _{t \rightarrow \infty} \Phi_{q}(t)$, often referred to as a glass form factor or Edwards-Anderson nonergodicity parameter. It describes the frozen-in structure of the glass and obeys

$$
\frac{f_{q}}{1-f_{q}}=\mathcal{F}_{q}[f] .
$$

In the fluid regime density fluctuations at different times decorrelate, so that the long time limit vanishes, $f_{q} \equiv 0$. On approaching a critical packing fraction $\phi_{c}$ or a critical temperature $T_{c}$, MCT states that strongly coordinated movements are necessary for structural rearrangements to relax to equilibrium. MCT identifies two slow structural processes, $\beta$ and $\alpha$ processes, when the glassy structure (described by $f_{q}^{c}$ ) becomes metastable and takes a long time to relax. In the idealized picture of MCT, dynamical arrest sets in at the glass transition with $f_{q}>0$ when the particles are hindered to 
escape from their neighboring environments. This also is accompanied by diverging relaxation times. Glassy states are called nonergodic states in MCT. The value of the glass form factor at the transition is called critical nonergodicity parameter $f_{q}^{c}$.

Although experiments on molecular glass formers have revealed that the dynamics very close to the transition point is dominated by thermally activated hopping processes, which the described (idealized) MCT cannot account for, MCT has been very successful in describing the approach to glassy arrest. It gives a quite accurate description of structural relaxation in colloidal dynamics and there especially of the $\alpha$ process.

For liquid states and large times the correlator approaches the $\alpha$-scaling law $\Phi_{q}(t) \rightarrow \widetilde{\Phi}_{q}(\widetilde{t})$ and $\tilde{t}=t / \tau$. Here the scaling functions $\widetilde{\Phi}_{q}(\widetilde{t})$ are independent of temperature or other control parameters. The $\alpha$-relaxation scale $\tau$ is given in MCT by

$$
\tau=\tau(\varepsilon)=\tau_{0} /|\varepsilon|^{\gamma} \quad \text { with } \quad \gamma=\frac{1}{2 a}+\frac{1}{2 b},
$$

and depends only on the separation $\varepsilon=\frac{T_{c}-T}{T_{c}}$ from the critical point. The scaling factor $\tau_{0}$ needs to be determined from matching the microscopic dynamics and the $\beta$-scaling regime to the $\alpha$-scaling regime. The anomalous exponents $a$ and $b$ are solutions of the equation

$$
\Gamma^{2}(1-x) / \Gamma(1-2 x)=\lambda
$$

for $0<x=a<1 / 2$ and $-1 \leq x=-b<0$. The exponent parameter $\lambda$ enters in the second order of the asymptotic expansion of the right-hand side of Eqs. (3) and (5) around the critical plateau $f_{q}^{c}$. Therefore it depends on the structure factor $S_{q}^{c}$ at the critical point via the vertex Eq. (4).

In the vicinity of the critical point, von Schweidler's power law describes the initial $\alpha$ relaxation from the plateau to zero. It is nothing more than the short-time expansion of the $\alpha$-master functions, which is up to second order

$$
\widetilde{\Phi}_{q}(\widetilde{t})=f_{q}^{c}-h_{q} \tilde{t}^{b}\left(1+k_{q} \tilde{t}^{b}\right)+O\left(\widetilde{t}^{3 b}\right) .
$$

The coefficients $h_{q}$ are called critical amplitude, the $k_{q}$ are correction amplitudes [43]. Von Schweidler's law is the origin of stretching (viz., nonexponentiality) in the $\alpha$ process of MCT.

The final decay of the structural relaxations in different correlators provides a definition of the $\alpha$-relaxation times. We use $\Phi_{q}\left(t=\tau_{q}\right)=f_{q}^{c} / 20$. In MCT the increase of the relaxation times in different correlators is strongly coupled. Their divergence is inherited directly from the diverging $\alpha$-relaxation scale $\tau$ of Eq. (8). For the $\alpha$-relaxation times of the density fluctuations in $\Phi_{q}(t)$ this means a separation into $\alpha$ scale and a dimensionless factor $\hat{t}_{q}$ containing the wavevector dependence.

$$
\tau_{q}=\hat{t}_{q} \tau(\varepsilon) .
$$

For large wave vectors, $\hat{t}_{q} \sim q^{-1 / b}$ holds in MCT [44].

Summarizing this short presentation of MCT, let us note that the wave-vector dependences of the various amplitudes in the asymptotic MCT predictions will enable us in the fol- lowing to identify the physical mechanisms causing glassy arrest. More details about MCT, the asymptotic expansions, and the scaling laws can be found in $[41-43,45]$.

\section{SIMULATION SETUP}

Molecular dynamics simulations in the canonical ensemble were performed considering 1000 quasihard particles interacting by a short-range attraction. Because we aim to study the fluid-to-nonergodic transition induced by attractions, equilibrium phase transitions, i.e., crystallization and liquid-gas separation, were suppressed by suitable choices for the interaction potential, which we introduce in the following.

The short-range attraction mimics the interaction between colloidal particles induced by non-adsorbing polymers in a colloid polymer mixture. For monodisperse colloids, this attraction is given by the Asakura-Oosawa interaction [1], which is slightly modified to include polydispersity [46]. The attraction strength is set by the concentration of polymers, $\phi_{p}$, and the range by the polymer size, $\xi$ (see below). This potential has been slightly corrected near contact $r=d_{12}$ to ensure that the total interaction potential has the minimum at $d_{12}\left[d_{12}=\left(a_{1}+a_{2}\right)\right.$, with $a_{1}$ and $a_{2}$ the radii of the interacting particles] [31].

Crystallization is avoided by using a polydisperse system: particle sizes are distributed according to a flat distribution of width $\delta=0.1 a$, where $a$ is the mean radius. The core-core repulsion is given by $V_{s c}(r)=k_{B} T\left(r / d_{12}\right)^{-36}$.

At high polymer fractions, or attraction strength, this system separates into two fluid phases with different densities, dilute and dense- the critical point is at $\phi_{p}^{\mathrm{cp}} \approx 0.29$. To avoid this transition, which would interfere with the attractive glass, a repulsive long-range barrier has been added to the total interaction potential, which extends from $r=d_{12}+2 \xi$ to $r=4 a$, and its height is only $1 k_{B} T$ (equal to the attraction strength at $\left.\phi_{p}=0.0625\right)$. The barrier raises the energy of a dense phase, so that liquid-gas separation does not take place. The resulting total interaction potential $V_{\text {tot }}=V_{\mathrm{sc}}$ $+V_{\mathrm{AO}}+V_{\mathrm{bar}}$ is analytical everywhere and allows straightforward integration of the equations of motions. The total interaction potential is presented in Fig. 1 for particles with the average radius.

The inclusion of the repulsive barrier in the interaction potential effectively inhibits liquid-gas separation [31], but causes holes and tunnels in the system. This is reflected in the structure factor as a low- $q$ peak, which grows and moves to lower- $q$ values, as the strength of the attraction (namely, $\phi_{p}$ ) increases. The effect of this barrier on the glass transition is studied below within MCT.

Lengths are measured in units of the average radius $a$, time in units of $\sqrt{4 a^{2} / 3 v^{2}}$, where the thermal velocity $v$ was set to $\sqrt{4 / 3}$. Equations of motion were integrated using the velocity-Verlet algorithm, in the canonical ensemble (constant $N T V$ ), to mimic the colloidal dynamics. Every $n_{t}$ time steps, the velocity of the particles was rescaled to assure constant temperature. No effect of $n_{t}$ was observed for well equilibrated samples. The time step was set to 0.0025 . The range of the attraction $2 \xi$ is set to $2 \xi=0.2 a$. The density of 


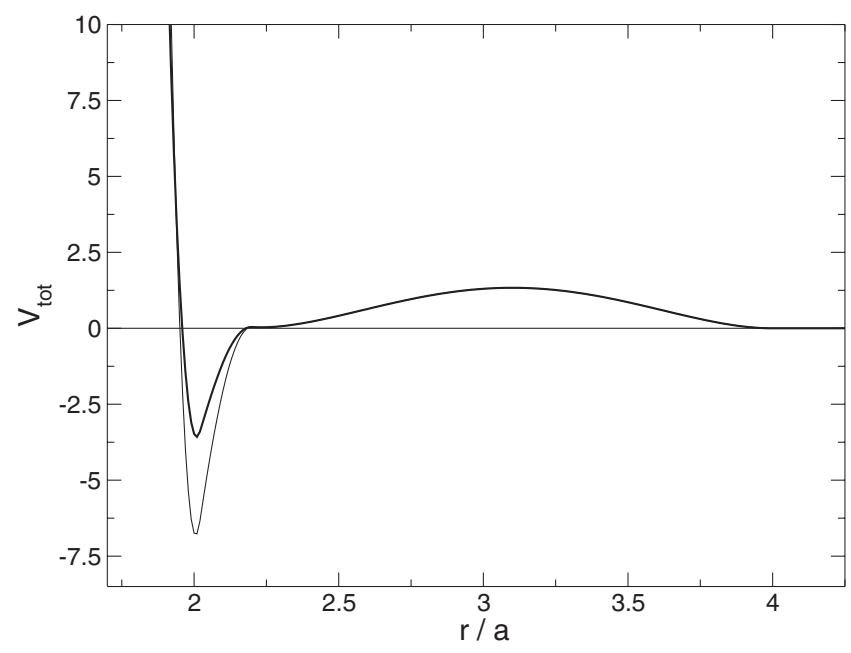

FIG. 1. Interaction potential for two particles with the average radius. The polymer fractions are $\phi_{p}=0.42$ and $\phi_{p}=0.25$ for the (lower) thin and (upper) thick curves, respectively (values close to the glass transition in the simulation and MCT, respectively). Note that in our units, the thermal energy is $k_{B} T=4 / 3$.

colloids is reported as volume fraction $\phi_{c}=\frac{4}{3} \pi a^{3}\left[1+\left(\frac{\delta}{a}\right)^{2}\right] n_{c}$, with $n_{c}$ the colloid number density, and the attraction strength is given by the polymer volume fraction $\phi_{p}$ (with $\xi=0.1$, the minimum of the attraction for average sized particles at $r=2 a$ is $\left.V_{\min }=-16 k_{B} T \phi_{p}\right)$.

The dynamics of this system has been analyzed previously within the framework of MCT, i.e., using the density correlation functions [31,32]. Increasing the attraction strength $\phi_{p}$ at $\phi_{c}=0.40$ a glass transition is obtained at $\phi_{p}^{c}=0.4265$, which shows the qualitative features of attractive glasses, as predicted by MCT. The critical parameters given below for the transition were obtained analyzing $\Phi_{q}(t)$ for the fluid state $\phi_{p}=0.42$, close enough to the glass to show the typical two-step decay (see Fig. 8)-1500 independent configurations were used to calculate $\Phi_{q}(t)$, from 15 independent "quenches" from hard spheres at the same density. The $\beta$ regime and early $\alpha$ decay were analyzed from $t=2$ to $t=500$. A wave-vector-independent von Schweidler exponent was fitted using the correlators at all wave vectors, whereas the nonergodicity parameter $f_{q}^{c}$ and critical amplitudes $h_{q}$ and $k_{q}$ were actually fitted for every wave vector.

The structure factors needed as inputs to MCT were calculated from the definition of $S_{q}$, using only the allowed wave vectors $\mathbf{q}=2 \pi / L\left(n_{x}, n_{y}, n_{z}\right)$, with $L$ the box size and $n_{x}$, $n_{y}$, and $n_{z}$ integers. Starting from $q=2 \pi / L$, the next value of the $\mathbf{q}$ modulus is selected if the $q$ separation is larger than $0.1 a^{-1}$, up to $q a=40$. The structure factors were then interpolated to have a constant $q$ grid.

\section{ASPECTS OF THE NUMERICAL MCT SOLUTIONS}

For the numerical solution of the MCT equations, algorithms were used that have been developed in the recent years [47]. Dynamic and static analyses were performed by iteratively solving Eq. (2) with the memory functional given by Eqs. (3) and (4). The results were accepted if a conver- gence to $\Phi_{q}(t)$ with a relative accuracy of $10^{-15}$ was achieved at each $t, q$. To extend the calculation onto logarithmic time scales without running into inefficient time discretization for late times, we used an algorithm for the convolution in Eq. (2) that doubled the initial time step of $10^{-9} D^{2} / D^{s}$ every 256 time steps, where $D=2 a$ is the particle diameter. The critical polymer concentrations were attained by a bisection method and determined up to a relative accuracy of $10^{-5}$ in concentration.

The structure factors for the MCT calculation input were taken directly from the simulation and linearly interpolated without further smoothing. We used a wave-vector grid with $M=400$ grid points and a cutoff of $q D=80$. From [48] it is known that with these values neither the discretization nor the cutoff influence the results significantly. For very small wave vectors $q D \leq 0.3$ the algorithm does not produce the correct results because of numerical error propagation. This can be recognized by the static results shown in Figs. 4 and 5 for the nonergodicity parameter $f_{q}$, in Fig. 6 for the critical amplitudes $h_{q}$, and in Fig. 7 for the $\alpha$-relaxation times. The plotted results exhibit a sudden drop down to 0 for $q \rightarrow 0$, whereas they should take a finite value. Despite this, the results for larger $q$ are not invalidated, as was verified by removing the incorrect values from the integrations in Eq. (3).

\section{RESULTS AND DISCUSSION}

In order to quantitatively describe the results from the simulations for the polydisperse system of particles interacting with the potential shown in Fig. 1, three different calculations within MCT were performed. They differ in the input static structure factors, all of which were obtained directly from simulations. Three additional simulation studies were performed solely to generate the $S_{q}$, where the pair potentials employed in the different simulations differed. Thus, we could highlight the importance of (i) particle polydispersity, and (ii) the long-ranged repulsive barrier, in the structural relaxation.

For convenience and clarity we name the different MCT calculations in the following way: System $(A)$ is the monodisperse model without repulsive barrier, whereas we refer to $(B)$ and $(C)$ as the monodisperse and polydisperse systems, respectively, with repulsive barrier. While the calculation in $(C)$ thus uses exactly the $S_{q}$ of the system whose dynamics we aim to describe, the MCT we use considers a monodisperse system. Calculation $(C)$ thus also is only approximative. True multispecies MCT calculations like in Ref. [49] would be required to capture all polydispersity aspects, yet are too demanding in the present case. All elements of the matrix of partial structure factors would be required, and the multiple wave-vector integrations over the required large $q$ ranges would crucially slow down the MCT numerics. In order to stress the approximative character of the MCT calculation $(C)$, we call the simulation, where the dynamics is analyzed, system $(D)$. Some results of the calculations such as critical polymer concentrations, exponent parameters, exponents, and localization length (in units of the diameter $D$ ) are summarized in Table I. 
TABLE I. Critical polymer concentrations, exponent parameters, von Schweidler exponents, and localization lengths resulting from the different MCT computations: $(A)$ monodisperse without barrier, $(B)$ monodisperse with barrier, $(C)$ polydisperse with barrier. The first columns give polydispersity $\delta$ and whether a repulsive barrier exists. Listed under $(D)$ are the corresponding parameters from the analysis to the polydisperse simulation with a barrier of the fluid state with $\phi_{p}=0.42$. All states are at colloid packing fraction $\phi_{c}=0.40$.

\begin{tabular}{lllcccl}
\hline \hline & $\delta$ & $V_{\text {bar }}$ & $\phi_{p}^{c}$ & $\lambda$ & $b$ & $r_{l}^{2}$ \\
\hline$(A)$ MCT & 0 & No & 0.2356 & 0.752 & 0.555 & 0.00501 \\
$(B)$ MCT & 0 & Yes & 0.2464 & 0.759 & 0.544 & 0.00510 \\
$(C)$ MCT & 0.10 & Yes & 0.3646 & 0.775 & 0.517 & 0.02628 \\
$(D)$ Simulation & 0.10 & Yes & 0.4265 & 0.863 & 0.37 & 0.0158 \\
\hline \hline
\end{tabular}

\section{A. Structure factors}

To clarify the influences of the different pair potentials and the polydispersity on the equilibrium structure we compare on the one hand structure factors for the same parameters $\left(\phi_{c}, \phi_{p}\right)$ for the different potentials and on the other hand, results at the MCT-critical points that are obtained for the different potentials. The $S_{q}$ in Fig. 2 for $\phi_{c}=0.40$ and $\phi_{p}=0.25$ all show a primary peak at $q D \approx 7.5$ that indicates the local fluid order. A peculiarity, which is often seen in gels of intermediate and lower densities, is a low- $q$ peak in the static structure factor. It appears on the length scale of the voids in the structure when the sol-gel transition line is reached, increases towards the transition $[6,50]$, and shifts to slightly lower $q$ values (cf. Fig. 3). A common interpretation of the low- $q$ peak is that it indicates the onset of an arrested phase separation at higher attraction strengths. At lower density, below the percolation threshold, this peak marks the presence of clusters in the system, although similar internal structures for gels and independent clusters have been re-

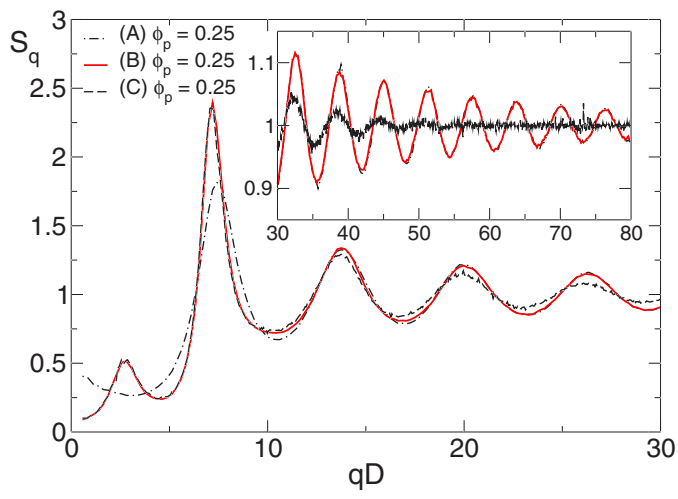

FIG. 2. (Color online) $S_{q}$ from MD simulations at a colloid packing fraction of $\phi_{c}=0.40$ and a polymer concentration of $\phi_{p}=0.25$, which is in the gel close to the critical point in MCT. The repulsive barrier affects $S_{q}$ in the region below $q D \leq 13$; system $(A)$ without barrier (dashed-dotted, black) shows neither a prepeak nor a primary peak, which is as high as in systems $(B)$ and $(C)$ with barrier (full red, dashed black). The inset demonstrates that polydispersity causes the $q$-tail oscillations in $S_{q}$ to be suppressed for large wave vectors. The $S_{q}$ for the polydisperse model $(C)$ falls below the noise level for $q D \geq 45$, whereas the monodisperse systems $(A)$ and $(B)$ virtually coincide there. ported [10]. The systems $(B)$ and $(C)$ with repulsive barrier show this low- $q$ peak, whereas it is absent in the model $(A)$ without repulsive barrier. The latter $S_{q}$ clearly grows in the limit $q \rightarrow 0$ for increasing polymer fractions, indicating the proximity of the liquid-gas critical point. The $S_{q}$ with barrier stay finite as a result of the weak repulsion preventing phase separation; rather they develop the prepeak, which may signal closeness to microphase separation.

Besides the long-ranged barrier, polydispersity has important effects on the equilibrium structure. The inset of Fig. 2 displays $S_{q}$ from $q D=30-80$ for the different systems. The $S_{q}$ of the monodisperse systems both with and without barrier virtually coincide and show distinct oscillations for these wave vectors, unlike in the polydisperse case. Indeed $S_{q}$ for $(C)$ starts to deviate from $(B)$ above $q D \approx 12$ and decays to the noise level above $q D \approx 45$. This rapid decay to unity in the $S_{q}$ of the polydisperse systems is due to slight differences in the distances where the (partial) pair correlation functions for differently sized particles show their contact maxima. This distribution in the contact distances leads to negative interferences in the oscillatory large- $q$ pattern in $S_{q}$, which gets canceled in the averaged structure factor of the polydis-

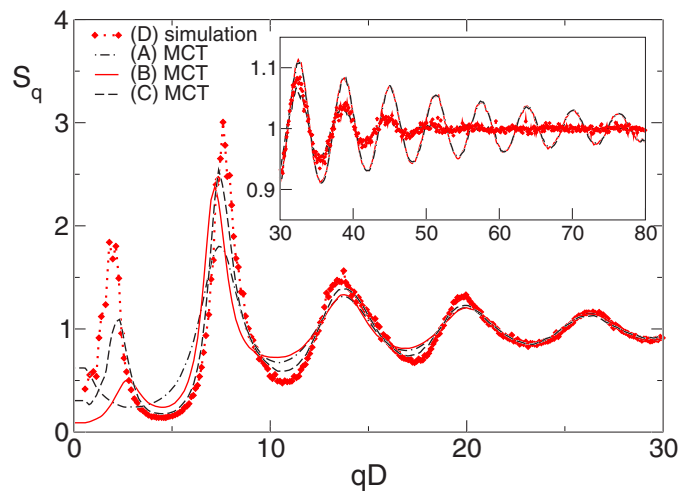

FIG. 3. (Color online) Critical $S_{q}$ at the boundaries of the gel phase: Black dashed-dotted, red full, and black dashed lines mark results from $(A),(B)$, and $(C)$. The red diamonds indicate $S_{q}$ at $\phi_{p}=0.42$, which is close to the arrested state in the simulation $(D)$. In the simulation, the prepeak at $q D \simeq 2.5$ rises and shifts to smaller $q$ values with increasing polymer concentration. The inset gives an enlarged view of the $q$ tail where systems $(A)$ and $(B)$ show pronounced oscillations driven by the short-ranged attraction. 
perse system. This effect of the short-ranged attraction, viz., the increased probability of particle contact, is thus only contained in the $S_{q}$ of systems $(A)$ and $(B)$.

Figure 3 shows $S_{q}$ at the critical points in MCT (see Table I) and in the simulation $\left[(D), \phi_{p}^{c} \approx 0.4265\right]$. It was possible to take the structure factors for the MCT input directly from the monodisperse simulations, because MCT predicts the states to be nonergodic already at rather low $\phi_{p}$, while the actual system only freezes at higher attraction strengths. The features discussed with respect to Fig. 2 can be recognized again even though the attraction strengths vary. While $S_{q}$ of the system $(A)$ without barrier differs from the other systems at small wave vectors, polydispersity forces the averaged $S_{q}$ of systems $(C)$ and $(D)$ to approach unity quickly at large wave vectors.

\section{B. Nonergodicity parameters}

From the equilibrium structure factor input of the different systems we calculated the critical Edwards-Anderson nonergodicity parameters $f_{q}^{c}$ using Eq. (5). The bifurcation occurs for the colloid packing fractions of $\phi_{c}=0.40$, which has been used throughout the analysis, at polymer concentrations $\phi_{p}^{c}=0.2356(A), \phi_{p}^{c}=0.2464(B)$, and $\phi_{p}^{c}=0.3646(C)$, respectively. Note that the critical polymer concentrations are lower than the critical $\phi_{p}^{c}$ in the simulation, system $(D)$. Luckily, the transition point in system $(A)$ occurs for attractions lower than the critical point, which suppresses effects from the liquid-gas separation. For system $(B)$, crystallization is far too slow to affect the results (although this state is indeed metastable with respect to crystallization). The trend of MCT to overestimate the tendency to freeze leads almost to a factor of 2 in terms of attraction strengths [48]. This discrepancy has also been observed in former comparisons of MCT with binary Lennard-Jones fluids [35], taking the $S_{q}$ from simulations. Similarly, a factor of approximately 2 in the temperature axis has been observed close to the merging of attraction- and repulsion-driven glass lines in a binary mixture [29]. A bilinear transformation of density and temperature was proposed to map the MCT calculations using Percus-Yevick $S_{q}$ onto the estimated transition points from simulations at high density [29].

The nonergodicity parameter $f_{q}^{c}$ basically oscillates in phase with the $S_{q}$. The shape of $f_{q}^{c}$ serves to identify the leading mechanism for the freezing. A repulsion-driven transition creates an $f_{q}^{c}$ with pronounced peaks and lower values for small wave vectors. Characteristically, it decreases quickly to zero for increasing $q$. The width of $f_{q}$ as a function of $q$ can be taken as a measure for the localization length that describes the spatial extent that a single particle can explore within its glass cage. For repulsion-driven glass transitions one generally finds a localization length of the order of the Lindemann-length, viz., a value around a tenth of the average particle separation [42]. If attraction drives the transition, the critical nonergodicity parameters have higher values and smaller localization lengths, showing up in much wider $f_{q}^{c}$ distributions than for repulsion-driven nonergodic states. The width of $f_{q}$ as a function of $q$ is now set by the attraction range. In our analysis $f_{q}^{c}$ compares quite well with the simu-

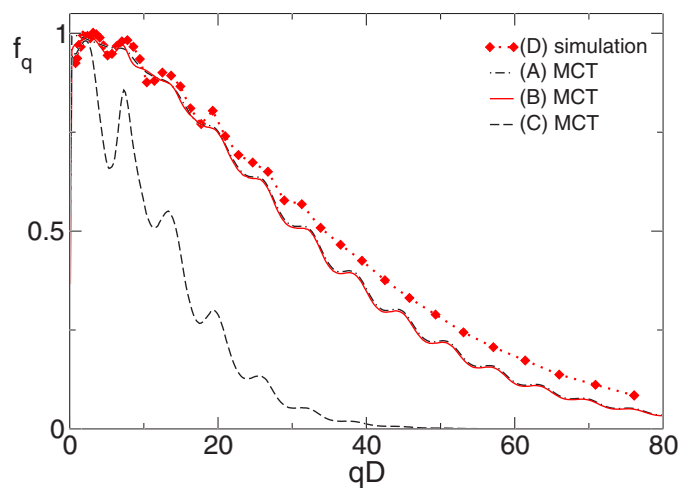

FIG. 4. (Color online) Critical nonergodicity parameters $f_{q}^{c}$ at the transition in the simulation $(D)$ (red diamonds), from MCT with monodisperse $[(A),(B)] S_{q}$ (black dashed-dotted and red full line) and polydisperse $(C) S_{q}$ (black dashed line). Serious differences occur, when the average $S_{q}$ of a polydisperse system is used as input to a monodisperse theory; the shape of $f_{q}$ for $(C)$ resembles more one of a repulsive glass.

lation results for those models where an attraction-driven large- $q$ tail is present in $S_{q}$ (see Fig. 4). The MCT calculation for the polydispersity-smeared-out $S_{q}$ gives far too small $f_{q}^{c}$ and too large localization lengths which resemble more results from repulsion-driven systems. We conclude from the agreement between the $f_{q}^{c}$ from simulation and the MCT calculations with attraction-driven large- $q$ tails in $S_{q}$, that the simulations exhibit an attraction-driven glass transition or what could be referred to as a gel. Note that this good agreement is found despite the overestimation of the critical attraction, i.e., the transition is wrongly located but its principal property is correctly predicted.

An important point to be checked is the role of the longranged repulsive barrier. We have already stated above that there is hardly any difference between the critical polymer concentrations in the monodisperse calculations without $(A)$ and with barrier $(B)$. Figure 5 highlights the influence of the barrier on the nonergodicity parameters $f_{q}^{c}$. The localization

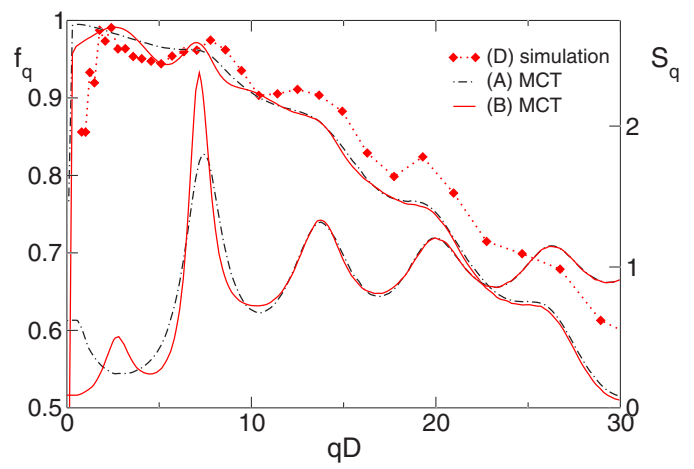

FIG. 5. (Color online) Enlarged view of the nonergodicity parameters of Fig. 4 and structure factors in the low- $q$ region: Minor differences in $f_{q}^{c}$ show up in the region $q D \leq 10$ only, where also the input $S_{q}$ differs considerably. Simulation data $[(D)$ red symbols] agree qualitatively with the model calculation $(B)$ including the barrier. 


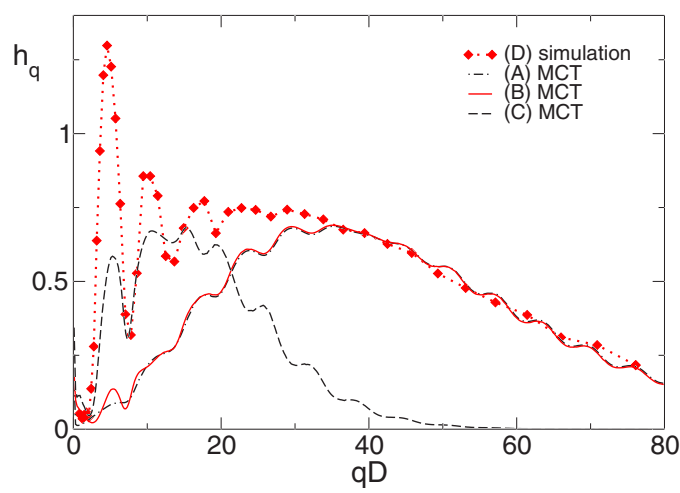

FIG. 6. (Color online) Critical amplitudes in simulation $(D)$ (red diamonds) and MCT $[(A),(B),(C)]$ (black dashed-dotted, red full, black dashed) close to dynamic arrest. Because of the different $\alpha$ times in simulation and theory the MCT results for $(A),(B)$ were scaled on the simulation results.

length as well as the attraction-driven character of the glass remain unchanged. A significant effect could only be observed in the vicinity of the prepeak and the primary peak, where $S_{q}$ also changes. Domains of higher wave vectors stay practically unaffected (see also Fig. 4). This indicates that the modes on the low angle $q$ peak, which is related to the void structure seen in the simulations, follow the relaxation of an attraction-driven glass without dominating it. It is dominated solely by large- $q$ modes.

\section{Critical amplitudes and von Schweidler law}

Asymptotic expansions of Eq. (5) around the critical plateau $f_{q}^{c}$ introduce the critical amplitude $h_{q}$ in linear order of the so-called $\beta$ process. During the $\beta$ process, the dynamics on all length scales is strongly coupled. The amplitude $h_{q}$ measures the participation of the correlator at wave vector $q$ in this process. The $\beta$ process describes the rate limiting process of glassy arrest, as here glassy and fluid dynamics start to deviate. The amplitude $h_{q}$ thus provides important information on the physical mechanism causing glassy arrest. It generally exhibits a minimum as a function of $q$ at the maximum of $f_{q}^{c}$, which describes the structure that gets frozen in at the glass transition.

From simulation data, $h_{q}$ can be obtained via von Schweidler fits to correlators. Table I reveals that in the present case these fits require somewhat smaller critical exponents $b$ compared to all MCT calculations $[A-C]$. This is consistent with the observation, see below in Sec. V E, that the relaxation appears to be somewhat more stretched in the simulation than predicted by MCT.

The critical amplitudes in Fig. 6, which are associated with the calculations where the attractions dominate $[A-B]$, all exhibit a very broad peak in $q$. This shows that very local motion takes part in the $\beta$ relaxation of an attraction-driven glass transition. The presence [in $(B)]$ or absence [in $(A)]$ of the prepeak does not influence $h_{q}$ beyond tiny changes for $q D<10$. MCT thus correctly identifies local bond formation as the rate limiting step during the $\beta$ process. MCT underestimates $h_{q}$ in the $q$ range below $q D=30$ and thus overesti- mates the stability of the glassy structure on intermediate and long length scales. von Schweidler's law, $\Phi_{q}(t)=f_{q}^{c}$ $-h_{q}(t / \tau)^{b}$ from Eq. (8), gives a much stronger initial relaxation of the frozen-in structure for $q D \leq 30$ in the simulations than in the MCT calculations. In the simulation the small- $q$ modes decay with a larger amplitude during the $\beta$ process. The overestimation of the stability of the incipient glassy structure on length scales larger than corresponding to the average particle position indicates that MCT misses some of the larger-distance relaxation mechanisms. Nevertheless, the possibility to match a common von Schweidler series Eq. (8) to the correlators at large and small wave vectors [31] supports our conclusion from Sec. V B that the structural relaxation for all $q$ is enslaved to local bond formation.

The underestimation of $h_{q}$ at low $q$ suggests that MCT underestimates the contribution of the repulsion-driven mechanism of vitrification in the present system. Apparently, in the simulated system attraction-driven and repulsiondriven glass transition compete, and both transition lines are close. Our quantitative MCT calculations (erroneously) position the system too far from the repulsive transitions. Within MCT, a higher order singularity appears in the vicinity of the merging of the two glass transition lines, signaled by $\lambda=1$. Indeed, a larger $\lambda$ exponent is observed in the simulations compared to the MCT calculations, which glassify due to bond formation solely.

\section{D. $\alpha$-relaxation times}

The criterion for quantitatively defining the $\alpha$-relaxation times is somewhat arbitrary. We chose the definition

$$
\Phi_{q}\left(\tau_{q}\right)=X f_{q},
$$

for the $\alpha$-relaxation times, where in the theory $X=0.05$ and in the simulation $X=0.50$. The latter choice was required because of the limited simulation time, but incurs larger corrections to the values of $\tau_{q}$ arising from faster relaxation processes. The different definitions are reconciled in the following comparison by normalization of the times $\tau_{q} / \tau_{q D=22.7}$, which brings out the $q$ dependence. The $\alpha$-relaxation times $\tau_{q}$ generally vary in phase with the nonergodicity parameter $f_{q}^{c}$ and the structure factor $S_{q}$, a phenomenon often referred to as de Gennes narrowing. Repulsion-driven glass transitions display the largest $\tau_{q}$ at the principal peak in $S_{q}$, which indicates that the cage formed by the particle's next neighbors induces the dynamical arrest. Here on the contrary, the slowest relaxation takes place either (i) at the prepeak, when the barrier causes void formation, or (ii) for $q \rightarrow 0$ in model $(A)$ without barrier on approaching the phase separation region.

Figure 7 gives $\alpha$-relaxation times calculated from the above definition and normalized to their value at $q D=22.7$. In MCT for models with barrier $[(B),(C)]$ one finds that the slowest modes are connected with the prepeak. In model $(A)$ $\tau_{q}$ decreases by more than a factor 2 at this $q$, when the prepeak in $S_{q}$ is eliminated. The simulations, however, do not allow one to check this difference, but only show that the slowest modes are those with $q D<2$. Nevertheless, the different dynamics at small $q$ has no further impact on the dynamics at larger $q$; in the inset of Fig. 7 the $\tau_{q}$ for all models 


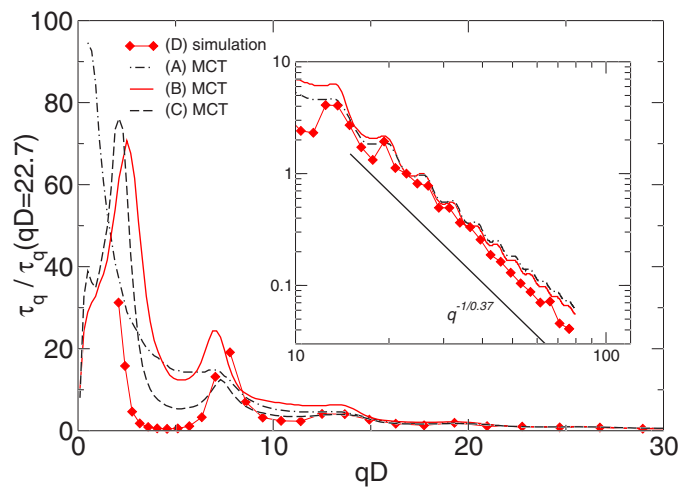

FIG. 7. (Color online) $\alpha$-relaxation times $\tau_{q}$ in MCT for the systems $(A),(B),(C)$ (black dashed-dotted, red full, black dashed line) and in the polydisperse simulation $[(D)$ red diamonds]. The curves are normalized to the value of $\tau_{q}$ at $q D=22.7$. In the inset, the results for models $(A)$ and $(B)$ and simulation $(D)$ are shown in a log-log plot. The straight line gives the asymptotic behavior $\tau_{q}$ $=q^{-1 / b}$ valid for large $q$ with the corresponding von Schweidler $b$ $=0.37$ obtained previously.

agree at large $q$, where the power law $\tau_{q} \sim q^{-1 / b}$ is also tested. It holds nicely in the simulation data, with the von Schweidler exponent obtained from the fitting of the correlation functions: $b=0.37$. MCT explains $\tau_{q}$ for larger wave vectors quite well, though small systematic deviations emerge because of the difference in the von Schweidler exponents between simulation and calculations, models $(A),(B)$, and $(C)$. Let us note in passing that the asymptotic behavior $\tau_{q}$ $\sim q^{-1 / b}$ holds earlier in the polydisperse MCT calculation $(C)$ (not shown), than in the monodisperse ones $(A)$ and $(B)$, where deviations are still noticeable in the inset of Fig. 7. This appears to support the probabilistic interpretation of the Kohlrausch law within MCT [42,44].

We conclude that the void structure is completely enslaved by the bond formation on local length scales. Even though the dissolution of the void structure is the slowest process, there is no evidence for a significant influence of the voids on the local dynamics. Local bond formation proceeds identically in systems $(B),(C),(D)$ with barrier and void prepeak in $S_{q}$, and in system $(A)$ without barrier and voidcorrelation peak in $S_{q}$.

\section{E. Correlation functions}

For an inclusive check of the results beyond asymptotic expansions we compare MCT correlators for a finite distance from the critical point obtained as full solutions to Eqs. (2)-(4). The fact that in simulations only finite distances from the transition point are accessible necessitates this comparison. We used the monodisperse model $(B)$, because it provides reasonable nonergodicity parameters and contains the barrier like the simulation $(D)$. The wave vectors for the MCT correlators have been chosen to be as close as possible to the simulation values $(\Delta q D \pm 0.1)$. The separation parameter for the MCT calculation was adjusted so that the structural relaxation can be compared most succinctly.

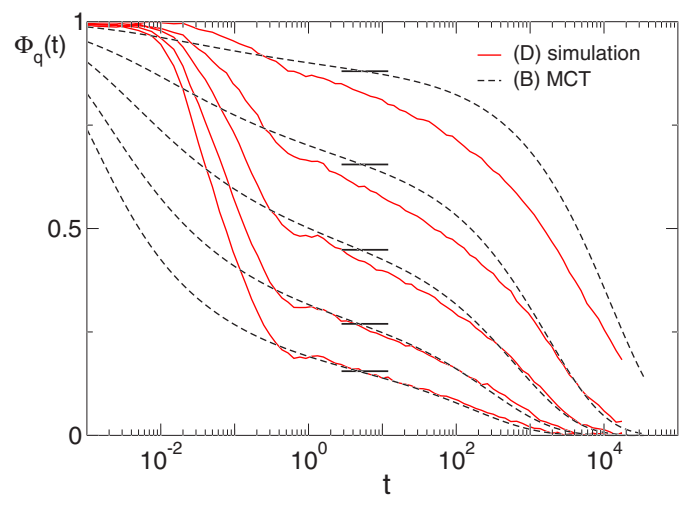

FIG. 8. (Color online) Density correlation functions in simulation $[(D)$ red solid lines] and MCT calculations in model $(B)$ (black dashed lines): The horizontal black bars indicate the critical plateau values of $f_{q}^{c}$ in MCT. The wave vectors from bottom to top are $q D=57.1,45.9,33.9,22.7,12.5$. The short-time diffusion coefficient was set to $D^{s} / D^{2}=0.133$. The theoretical curves correspond to a polymer concentration of $\phi_{p}=0.2461$, which means a separation parameter of about $\varepsilon_{\mathrm{MCT}}=-0.001$. The simulation results were obtained for a polymer concentration $\phi_{p}=0.42$, which corresponds to a separation parameter of $\varepsilon_{\text {simu }}=-0.015$.

The correlators from simulation in Fig. 8 show a two-step relaxation process with the final $\alpha$ relaxation from the plateau of height $f_{q}^{c}$. The $\beta$ process describes the dynamics close to $f_{q}^{c}$ including some part of the approach to $f_{q}^{c}$ from above. The simulation data exhibit damped vibrational motion on short time scales. This is neglected in Eq. (2), which therefore can describe the dynamics only at later times. It is only this structural relaxation that MCT addresses and thus the modeling at short times is done as simply as possible. We do not attempt to (a) include vibrational motion, (b) capture the separation of short-time and long-time dynamics quantitatively, but (c) only consider the shape of the structural relaxation in the following comparison.

The results highlight the strong $q$ dependence of the structural relaxation. In addition a strong stretching, i.e., nonexponential $\alpha$ relaxation, is also observed. According to different von Schweidler exponents of $b=0.37$ in the simulation and $b=0.54$ in MCT the simulation results appear somewhat more stretched. Nevertheless, the local dynamics, where the bond formation can be directly seen, is well described by MCT. Amplitude and shape of the $\alpha$ process are rather well captured, as would become even clearer if simulations closer to the transition could be performed. But for the dynamics on larger scales only qualitative statements can be made.

\section{CONCLUSIONS}

Glass transitions within MCT are bifurcation points in the equations of motion of the structural relaxation. While the equilibrium structure of the considered glass-forming fluid changes smoothly, the dynamics slows down significantly, and a metastable glassy structure comes into existence. The bifurcation transitions of MCT contain universal signatures, such as von Schweider's law that is the origin of the nonexponentiality of the (final or $\alpha$-) structural relaxation. Nonuni- 
versal amplitudes, such as the critical glass form factor $f_{q}^{c}$ or the critical amplitude $h_{q}$ entering von Schweidler's law, contain the information in MCT about the physical mechanisms causing arrest.

We considered the wave-vector dependence of $f_{q}^{c}, h_{q}$, and of the $\alpha$-relaxation times $\tau_{q}$ in order to discover the origin of glassy arrest in colloidal dispersions of particles with shortranged attractions at intermediate packing fractions. The study was motivated to gain insight into the connection between attraction-driven glass transitions at higher densities and colloidal gelation at lower ones. We looked at simulations of a model system where particles interact additionally with a weak long-range repulsive barrier.

Structure factors $S_{q}$ directly taken from simulations were used as the only input to MCT, in order to study the importance of the mesoscale gel-like structure indicated by a prepeak in $S_{q}$. It arises in the simulations from the long-ranged repulsive barrier that suppresses gas-liquid demixing. This mesoscale peak is the slowest mode in the system, as the void structure takes the longest time to dissolve. Yet, we find that the existence of the prepeak in $S_{q}$ does not affect MCT calculations for the attraction-driven glass transition. The latter is caused by local bond formation apparent in all quantities at large wave vectors. We conclude that the mesoscopic mode is enslaved to the formation of physical bonds, and that this local process is not affected by the larger-scale heterogeneities of the system. MCT quantiatively captures local bond formation but somewhat overestimates the stability of the glass on larger length scales. Still, the mechanism of arrest in the dispersion at intermediate density is the attraction-driven one discovered in MCT at higher densities. At lower density, the low- $q$ modes come into play and the properties of the glass transition discussed here are expected to change [24].

The role of polydispersity in MCT calculations was also considered. Averaged structure factors of polydisperse systems miss the large $q$ tail indicative of short-ranged attractions. This arises from negative interference of the various contributions from particle pairs with different contact distances. While this prevents the use of averaged $S_{q}$ from polydisperse systems to capture an attraction-driven glass transition, it does not imply that the vitrification mechanism in polydisperse systems is different. Rather the actual polydisperse system exhibits bond formation, and can be described quantitatively within MCT using the appropriate $S_{q}$ from, for example, the corresponding monodisperse system.

\section{ACKNOWLEDGMENTS}

We thank M. Cates for valuable discussions. This work was supported by "Acciones Integradas Hispano-Alemanas" of the DAAD, by the DFH/UFA, the IFPRI initiative "Gelling Systems," and by the DFG Grants No. SFB 513, International Research Training Group No. 667, and No. SP/ 714-3. A.M.P. acknowledges financial support from the MEC under project No. MAT2006-13646-C03-02, and J.B. acknowledges financial support by the IUF.
[1] W. C. K. Poon, J. Phys.: Condens. Matter 14, R859 (2002).

[2] P. N. Pusey and W. van Megen, Nature (London) 320, 340 (1986).

[3] K. N. Pham, A. M. Puertas, J. Bergenholtz, S. U. Egelhaaf, A. Moussaïd, P. N. Pusey, A. B. Schofield, M. E. Cates, M. Fuchs, and W. C. K. Poon, Science 296, 104 (2002).

[4] K. N. Pham, S. U. Egelhaaf, P. N. Pusey, and W. C. K. Poon, Phys. Rev. E 69, 011503 (2004).

[5] T. Eckert and E. Bartsch, Phys. Rev. Lett. 89, 125701 (2002).

[6] P. N. Segrè, V. Prasad, A. B. Schofield, and D. A. Weitz, Phys. Rev. Lett. 86, 6042 (2001).

[7] H. Sedgwick, S. U. Egelhaaf, and W. C. K. Poon, J. Phys.: Condens. Matter 16, S4913 (2004).

[8] P. J. Lu, J. C. Conrad, H. M. Wyss, A. B. Schofield, and D. A. Weitz, Phys. Rev. Lett. 96, 028306 (2006).

[9] F. Sciortino, A. Belloni, and P. Tartaglia, Phys. Rev. E 52, 4068 (1995).

[10] V. Gopalakrishnan and C. F. Zukoski, Phys. Rev. E 75, 021406 (2007).

[11] L. Fabbian, W. Götze, F. Sciortino, P. Tartaglia, and F. Thiery, Phys. Rev. E 59, R1347 (1999).

[12] L. Fabbian, A. Latz, R. Schilling, F. Sciortino, P. Tartaglia, and C. Theis, Phys. Rev. E 60, 5768 (1999).

[13] J. Bergenholtz and M. Fuchs, Phys. Rev. E 59, 5706 (1999).

[14] J. Bergenholtz, M. Fuchs, and T. Voigtmann, J. Phys.: Condens. Matter 12, 6575 (2000).
[15] K. Dawson, G. Foffi, M. Fuchs, W. Götze, F. Sciortino, M. Sperl, P. Tartaglia, T. Voigtmann, and E. Zaccarelli, Phys. Rev. E 63, 011401 (2001).

[16] J. Bergenholtz, W. C. K. Poon, and M. Fuchs, Langmuir 19, 4493 (2003).

[17] B. Ahlström and J. Bergenholtz, J. Phys.: Condens. Matter 19, 036102 (2007).

[18] H. Verduin and J. K. G. Dhont, J. Colloid Interface Sci. 172, 425 (1995).

[19] S. A. Shah, Y. L. Chen, K. S. Schweizer, and C. F. Zukoski, J. Chem. Phys. 118, 3350 (2003).

[20] S. Manley, H. M. Wyss, K. Miyazaki, J. C. Conrad, V. Trappe, L. J. Kaufman, D. R. Reichman, and D. A. Weitz, Phys. Rev. Lett. 95, 238302 (2005).

[21] G. Foffi, C. de Michele, F. Sciortino, and P. Tartaglia, J. Chem. Phys. 122, 224903 (2005).

[22] A. Stradner, H. Sedgwick, F. Cardinaux, W. C. K. Poon, S. U. Egelhaaf, and P. Schurtenberger, Nature (London) 432, 492 (2004).

[23] W. C. K. Poon, A. D. Pirie, and P. N. Pusey, Faraday Discuss. 101, 65 (1995).

[24] J. Wu, Y. Liu, W. R. Chen, J. Cao, and S.-H. Chen, Phys. Rev. E 70, 050401(R) (2004).

[25] R. P. S. W. M. Gelbart, J. Chem. Phys. 110, 4582 (1999).

[26] M. Carpineti and M. Giglio, Phys. Rev. Lett. 68, 3327 (1992).

[27] E. Zaccarelli, I. Saika-Voivod, S. V. Buldyrev, A. J. Moreno, P. 
Tartaglia, and F. Sciortino, J. Chem. Phys. 124, 124908 (2006).

[28] P. I. Hurtado, L. Berthier, and W. Kob, Phys. Rev. Lett. 98, 135503 (2007).

[29] F. Sciortino, P. Tartaglia, and E. Zaccarelli, Phys. Rev. Lett. 91, 268301 (2003).

[30] E. Zaccarelli, G. Foffi, K. A. Dawson, S. V. Buldyrev, F. Sciortino, and P. Tartaglia, Phys. Rev. E 66, 041402 (2002).

[31] A. M. Puertas, M. Fuchs, and M. E. Cates, Phys. Rev. E 67, 031406 (2003).

[32] A. M. Puertas, M. Fuchs, and M. E. Cates, J. Phys. Chem. B 110, 13220 (2006).

[33] A. M. Puertas, M. Fuchs, and M. E. Cates, Phys. Rev. Lett. 88, 098301 (2002).

[34] A. M. Puertas, M. Fuchs, and M. E. Cates, J. Phys.: Condens. Matter 19, 205140 (2007).

[35] M. Nauroth and W. Kob, Phys. Rev. E 55, 657 (1997).

[36] E. Flenner and G. Szamel, Phys. Rev. E 72, 031508 (2005).

[37] A. Winkler, A. Latz, R. Schilling, and C. Theis, Phys. Rev. E 62, 8004 (2000).

[38] F. Sciortino and W. Kob, Phys. Rev. Lett. 86, 648 (2001).
[39] T. Voigtmann and J. Horbach, Europhys. Lett. 74, 459 (2006).

[40] A. Meyer, J. Horbach, W. Kob, F. Kargl, and H. Schober, Phys. Rev. Lett. 93, 027801 (2004).

[41] W. Götze, in Liquids, Freezing and Glass Transition, edited by J. P. Hansen, D. Levesque, and J. Zinn-Justin (North Holland, Amsterdam, 1991), Vol. Session LI (1989) of Les Houches Summer Schools of Theoretical Physics, pp. 287-503.

[42] W. Götze and L. Sjögren, Rep. Prog. Phys. 55, 241 (1992).

[43] T. Franosch, M. Fuchs, W. Götze, M. R. Mayr, and A. P. Singh, Phys. Rev. E 55, 7153 (1997).

[44] M. Fuchs, J. Non-Cryst. Solids 172-174, 241 (1994).

[45] W. Götze, J. Phys.: Condens. Matter 11, A1 (1999).

[46] J. M. Méndez-Alcaraz and R. Klein, Phys. Rev. E 61, 4095 (2000).

[47] M. Fuchs, W. Götze, I. Hofacker, and A. Latz, J. Phys.: Condens. Matter 3, 5047 (1991).

[48] M. Sperl, Phys. Rev. E 69, 011401 (2004).

[49] G. Foffi, W. Götze, F. Sciortino, P. Tartaglia, and T. Voigtmann, Phys. Rev. E 69, 011505 (2004).

[50] F. Sciortino, S. Mossa, E. Zaccarelli, and P. Tartaglia, Phys. Rev. Lett. 93, 055701 (2004). 